\title{
The Impact of Race in Male Breast Cancer Treatment and Outcome in the United States: A Population-Based Analysis of 4,279 Patients
}

\author{
Jacob Y. Shin, Lisa A. Kachnic, and Ariel E. Hirsch \\ Department of Radiation Oncology, Boston Medical Center and Boston University School of Medicine, 830 Harrison Avenue, \\ Moakley Building LL 100, Boston, MA 02118, USA \\ Correspondence should be addressed to Ariel E. Hirsch; ariel.hirsch@bmc.org
}

Received 14 June 2014; Revised 8 September 2014; Accepted 20 September 2014; Published 2 October 2014

Academic Editor: Ian S. Fentiman

Copyright (C) 2014 Jacob Y. Shin et al. This is an open access article distributed under the Creative Commons Attribution License, which permits unrestricted use, distribution, and reproduction in any medium, provided the original work is properly cited.

\begin{abstract}
The purpose of this study is to compare the racial differences in treatment and overall survival (OS) of male breast cancer (MBC) patients. Data were extracted from the NCI SEER database that included population-based registries from 1988 to 2010 and analyzed using SPSS 20.0. 4,279 MBC patients were identified. 3,266 (76.3\%) patients were White, 552 (12.9\%) Black, 246 (5.7\%) Hispanic, and $215(5.0 \%)$ Asian. Black patients were more likely to be diagnosed at younger age $(P<0.001)$, have advanced stage disease $(P=0.001)$, and be unmarried $(P<0.001)$ and less likely to undergo lymph node dissection $(P=0.006)$. When stratified by stage, there was no difference in receipt of primary treatment by race. The 5-year OS for White, Black, Hispanic, and Asian races was $73.8 \%, 66.3 \%, 74.0 \%$, and $85.3 \%(P<0.001)$. This significant worse 5 -year OS for Blacks persisted regardless of age, stage II or III disease, and grade 2 or 3 disease. On multivariate analysis, Black race was a significant independent prognostic factor for worse OS. Blacks were less likely to receive lymph node dissection of which patients may derive benefit, though we did not observe receipt of primary treatment, after stratifying for disease stage, to be an underlying factor contributing to racial outcome differences.
\end{abstract}

\section{Introduction}

In 2014, there will be an estimated 2,240 new cases of male breast cancer $(\mathrm{MBC})$ in the United States, accounting for approximately $1 \%$ of all breast cancers annually [1]. It is estimated that 410 of these cases will end in death [1]. Though a rare disease, a previous population-based analysis has shown that the incidence has significantly increased from 0.86 to 1.08 per 100,000 population from 1973 to 1998 , with incidence reaching as high as 1.24 per 100,000 man-years in $2000[2,3]$. Mortality and survival rates have been shown to improve significantly over time, but to a significantly lesser extent in males compared to their female counterparts [3]. Age, tumor size, and nodal status have been shown to impact outcome in MBC patients [3-6].

However, the impact of race on MBC survival is still somewhat conflicting [7-11], which is in contrast to that seen in female breast cancer in which Blacks have been shown to have worse survival than Whites $[12,13]$. Patients with
MBC are more likely to be Black compared to their female counterparts $(23 \%$ versus $16 \%, P=0.013)$ [14], and the incidence of $\mathrm{MBC}$ is approximately 1.6 times higher in Black men when compared to Whites (1.8 versus 1.1 per 100,00 manyears) [9]. Thus, further understanding of the differences in presentation, treatment, and outcome by race in $\mathrm{MBC}$ patients is warranted.

In this population-based analysis, we sought to update our understanding of the impact of race on survival in $\mathrm{MBC}$, analyzing differences in demographics, clinicopathologic characteristics, and treatment types to further define relationships between race and outcome.

\section{Methods and Materials}

2.1. Patient Population. Data was extracted from the Surveillance, Epidemiology, and End Results (SEER) database of the United States National Cancer Institute. It includes eighteen population-based registries representing approximately $28 \%$ 
of the U.S. population [15]. Our study population consists of 4,279 male patients whose first, primary malignancy was diagnosed as breast cancer between 1988 and 2010. Patients younger than 18 years, with unknown disease stage, or whose primary treatment type was unknown were excluded. Additionally, if race was other than White, Black, Hispanic, or Asian, further exclusions were made.

2.2. Statistical Analysis. SPSS 20.0 (Armonk, NY: IBM Corp.) was used to perform all data analyses. Demographic, clinicopathologic, and treatment data by race including proportional differences in age at diagnosis, year of diagnosis, primary treatment, stage of disease, tumor grade, and marital status were compared using the Chi-square test. KaplanMeier estimates were used to analyze overall survival (OS) and disease-specific survival (DSS). The OS endpoint was defined as time to death from the date of diagnosis of breast cancer. The DSS endpoint was defined as time to death with cause of death being breast cancer, from the date of diagnosis of breast cancer. The Cox regression multivariate analysis was used to identify independent predictors for OS. A two-sided $P$ value $<0.05$ was considered statistically significant.

\section{Results}

3.1. Patient and Treatment Characteristics. 4,279 MBC patients were identified and the patient and treatment characteristics are displayed in Table 1. The median age of the study population was 65 years (range: 23-99). Racial demographics included 3,266 (76.3\%) Whites, 552 (12.9\%) Blacks, 246 (5.7\%) Hispanics, and 215 (5.0\%) Asians. The majority of patients, 2,919 (68.2\%), were married. Pathology included $3,552(83.0 \%)$ patients with invasive ductal carcinoma, 145 (3.4\%) lobular carcinoma, 73 (1.7\%) mucinous carcinoma, 61 (1.4\%) papillary carcinoma, $9(0.2 \%)$ medullary carcinoma, and 439 (10.3\%) ductal carcinoma in situ (DCIS). According to the AJCC 2010 staging system, 1,314 (30.7\%) patients were stage I, 1,628 (38.0\%) stage II, 668 (15.6\%) stage III, and 230 (5.4\%) stage IV. The majority of patients, 2,738 (64.0\%), did not have nodal metastases.

Modified radical mastectomy was performed in 2,424 (56.6\%) patients, while 1,027 (24.0\%) underwent simple mastectomy, 511 (11.9\%) partial mastectomy, 166 (3.9\%) no treatment, 59 (1.4\%) primary radiotherapy (RT), 41 (1.0\%) radical mastectomy, and $22(0.5 \%)$ nipple-sparing mastectomy. Lymph node dissection was performed in 3,583 (83.7\%) patients, and $932(21.8 \%)$ received adjuvant RT.

Blacks were more likely to be diagnosed at a younger age when compared with Whites, Hispanics, and Asians (61.2\% versus $43.8 \%$ versus $59.8 \%$ versus $54.0 \%$; $P<0.001$ ). They were also less likely to be married compared to the other races (52.2\% versus $70.2 \%$ versus $67.1 \%$ versus $80.5 \%$; $P<0.001)$. Twenty-one percent of Blacks did not undergo lymph node dissection compared to $15.3 \%$ in Whites, $18.7 \%$ in Hispanics, and $15.8 \%$ in Asians $(P=0.006)$. A significantly higher proportion of Blacks were more likely to be diagnosed with advanced stage disease compared to the other races (stage III: $18.7 \%$ versus $15.2 \%$ versus $15.9 \%$ versus $14.0 \%$, stage IV: 9.1\% versus $4.8 \%$ versus $5.3 \%$ versus $4.2 \% ; P=0.001$ ).
Blacks were also less likely to have estrogen receptor (ER) and progesterone receptor (PR) positive disease when compared to Whites, Hispanics, and Asians (56.0\% versus $64.8 \%$ versus $56.9 \%$ versus $67.0 \% ; P<0.001)$. When removing DCIS patients, significant racial differences were still observed when comparing by age $(P<0.001)$, marital status $(P<$ $0.001)$, lymph node dissection $(P=0.001)$, stage of disease $(P=0.001)$, and ER/PR status $(P<0.001)$.

When stratified by stage, there was no difference in receipt of primary treatment with respect to race (Table 2).

3.2. Survival Outcome. The median follow-up for the whole patient cohort was 54 months (range: 0-275). The 5-year overall survival rate (OS) for the study population was $73.4 \%$. Patients $\geq 65$ years had significantly worse 5 -year OS when compared with those $<65$ years $(64.5 \%$ versus $83.2 \%$; $P<$ 0.001 ). There was a significant OS improvement seen over the time periods 1988-1993, 1994-1999, and 2000-2005 (68.9\% versus $72.0 \%$ versus $75.2 \% ; P=0.018$ ). Those who were married had significantly higher 5-year OS when compared with those divorced, single, widowed, or separated $(77.5 \%$ versus $68.9 \%$ versus $68.7 \%$ versus $46.0 \%$; $P<0.001$ ) (Table 3 ).

Patients who did not receive any treatment $(45.9 \%)$ or those who received primary RT $(31.8 \%)$ had the worst 5year OS when compared to any type of mastectomy $(P<$ 0.001 ). There was a significant survival benefit for those who underwent lymph node dissection compared to those who did not $(76.0 \%$ versus $61.0 \% ; P<0.001)$. No survival difference was seen between those who did or did not receive adjuvant RT $(P=0.738)$.

Patients with nodal metastases had significantly worse 5 year OS than those who did not $(66.8 \%$ versus $77.0 \% ; P<$ 0.001 ). Five-year OS by disease stage was as follows: $91.3 \%$ for DCIS (stage 0 ), $83.8 \%$ for stage I, $74.2 \%$ stage II, $55.5 \%$ stage III, and 19.4\% stage IV $(P<0.001)$. Five-year OS for tumor grades 1,2 , and 3 was $84.7 \%, 76.5 \%$, and $65.2 \%$, respectively $(P<0.001)$.

Blacks had significantly worse 5-year OS when compared with Whites, Hispanics, and Asians (66.3\% versus 73.8\% versus $74.0 \%$ versus $85.3 \%$; $P<0.001$ ) (Figure 1). Blacks also had worse 5 -year OS in those $<65$ years ( $74.3 \%$ versus $84.8 \%$ versus $80.9 \%$ versus $88.6 \% ; P<0.001)$ and in those $\geq 65$ years (53.1\% versus $65.1 \%$ versus $60.7 \%$ versus $81.6 \%$; $P=0.011$ ) compared to the other races (Figures 2(a) and 2(b)). In those with invasive ductal carcinoma, 5-year OS was significantly worse for Blacks $(62.1 \%$ versus $71.2 \%$ versus $69.2 \%$ versus 83.5\%; $P<0.001$ ). Of patients with $\mathrm{ER}+/ \mathrm{PR}+$ status, 5 -year OS was $66.1 \%$ for Blacks versus $73.2 \%$ for Whites, $74.3 \%$ for Hispanics, and $89.6 \%$ for Asians $(P=0.006)$.

Blacks had significantly worse 5 -year OS compared to Whites, Hispanics, and Asians in those who underwent modified radical mastectomy $(64.9 \%$ versus $74.9 \%$ versus $71.6 \%$ versus $87.2 \% ; P=0.002)$, lymph node dissection (68.3\% versus $76.3 \%$ versus $75.7 \%$ versus $89.6 \% ; P<0.001$ ), and adjuvant RT (64.4\% versus $75.0 \%$ versus $67.9 \%$ versus $95.7 \% ; P=0.003$ ). In those with nodal metastases, Blacks also had significantly worse 5 -year OS compared to the other races (53.3\% versus $67.7 \%$ versus $69.6 \%$ versus $85.4 \%$; $P<0.001$ ). Of stage II patients, 5 -year OS was $67.0 \%$ in Blacks, $74.2 \%$ in 


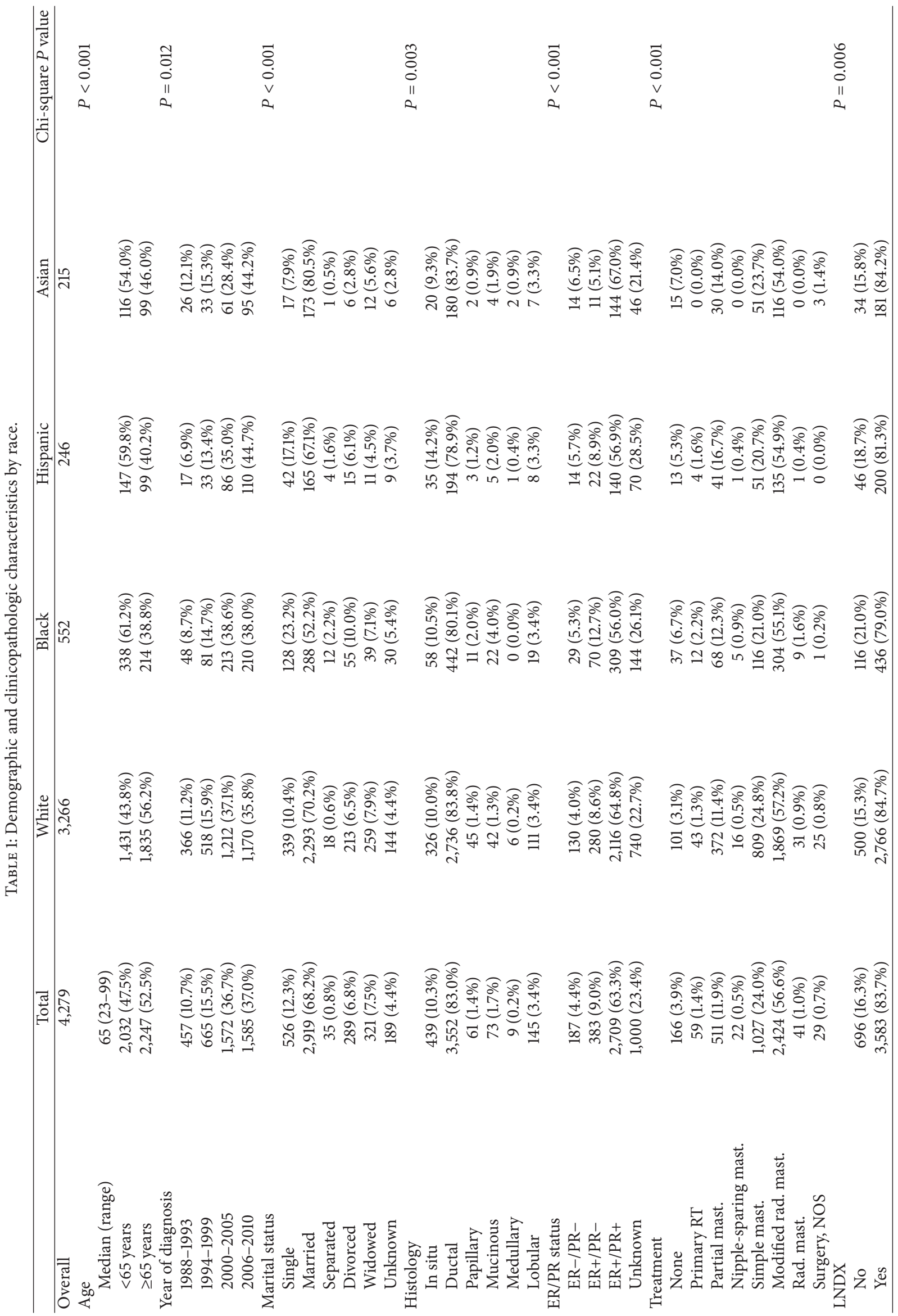




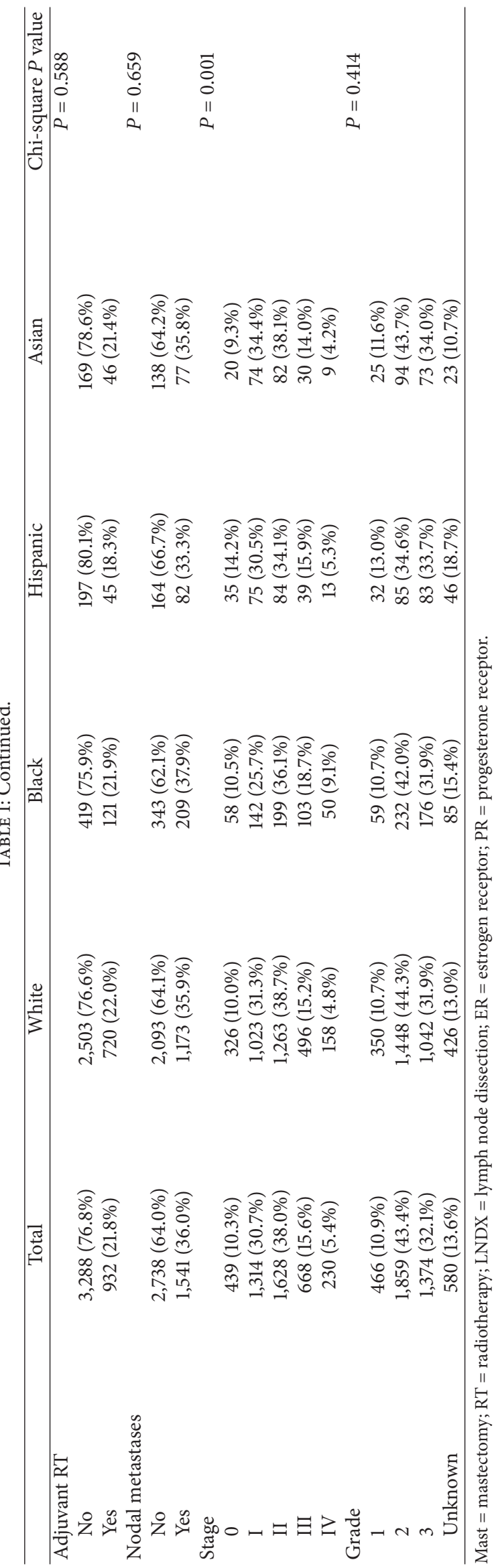




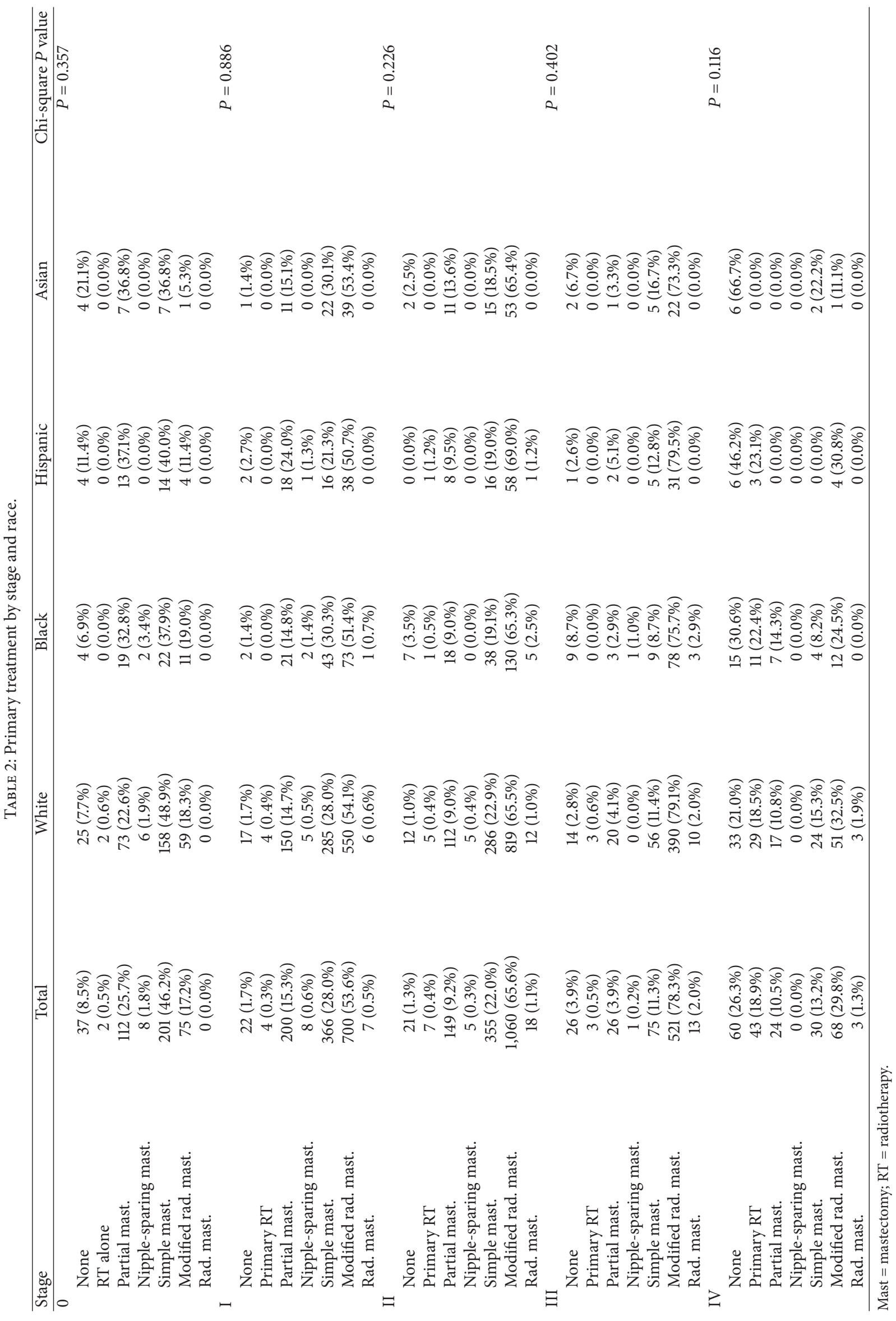




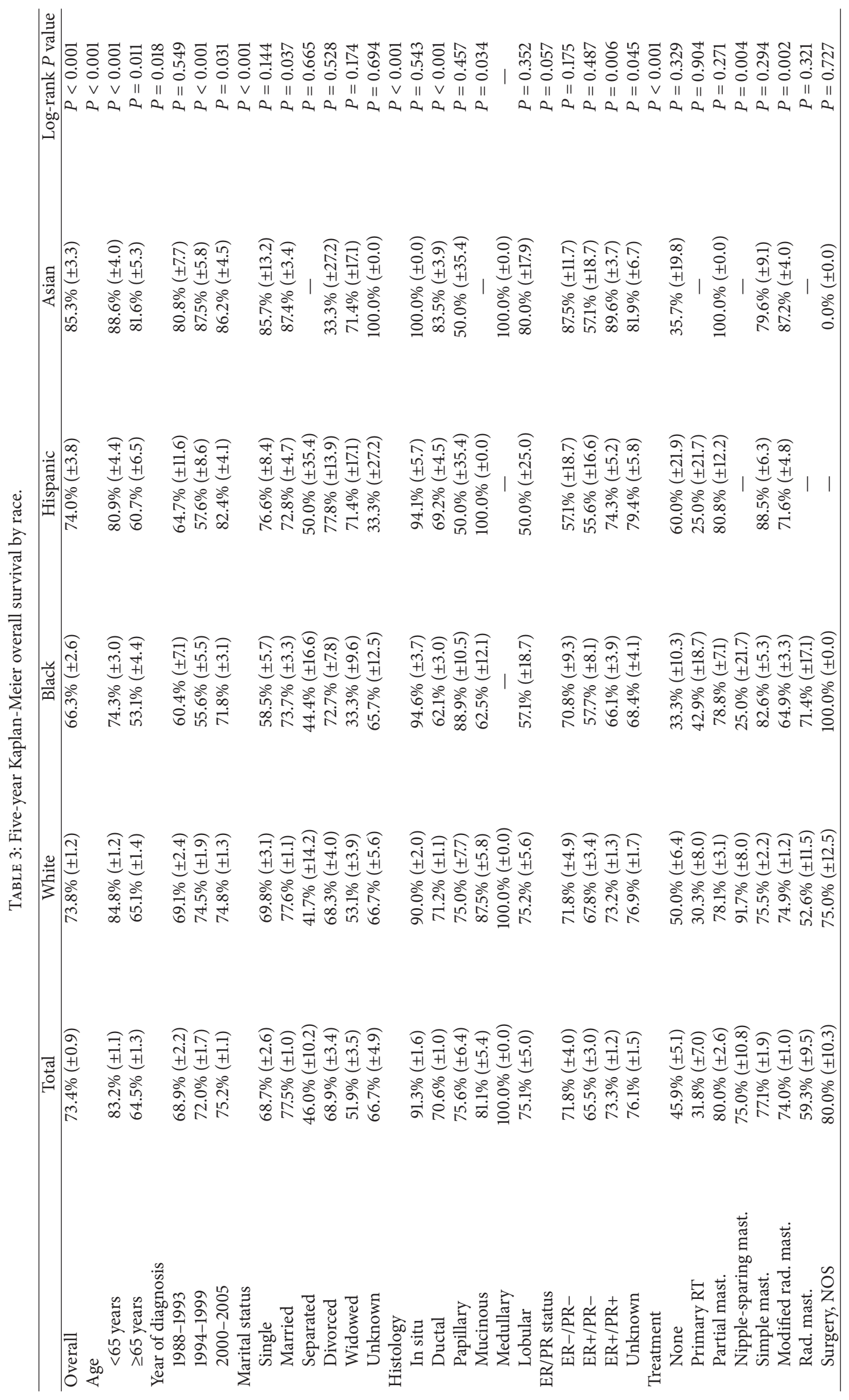




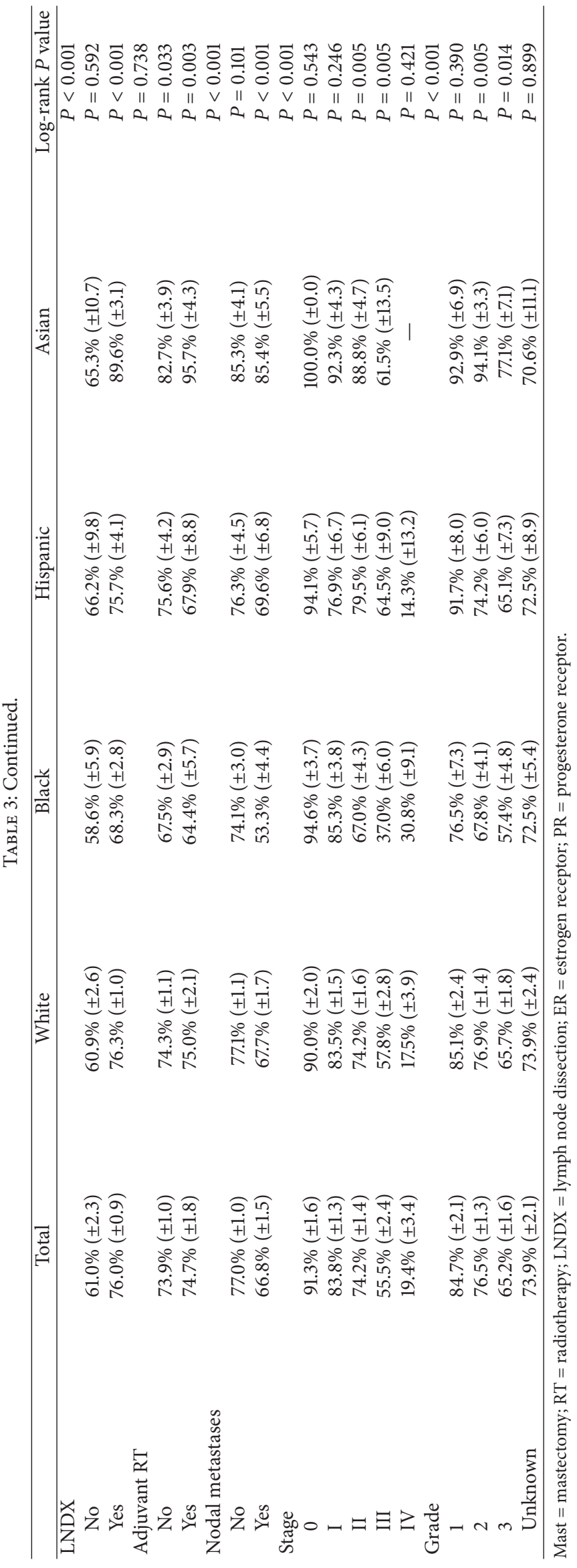




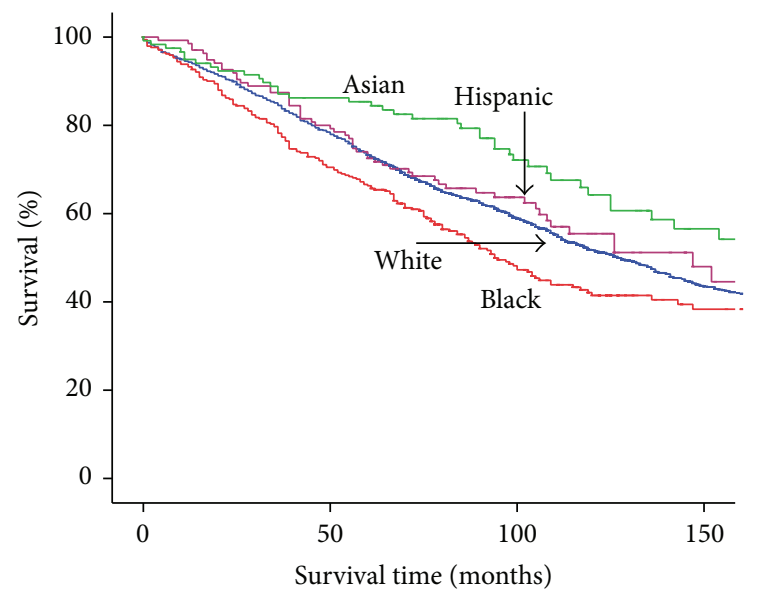

Numbers at risk

$\begin{array}{lllll}\text { White } & 2,096 & 1,636 & 835 & 310 \\ \text { Black } & 342 & 236 & 105 & 35 \\ \text { Hispanic } & 136 & 106 & 52 & 14 \\ \text { Asian } & 120 & 98 & 54 & 24\end{array}$

FIGURE 1: Kaplan-Meier overall survival by race.

Whites, $79.5 \%$ in Hispanics, and $88.8 \%$ in Asians $(P=0.005)$. Five-year OS was also significantly worse in Blacks compared to the other races among stage III patients (37.0\% versus $57.8 \%$ versus $64.5 \%$ versus $61.5 \% ; P=0.005)$. Finally, this survival disadvantage for Blacks compared to Whites, Hispanics, and Asians persisted in those with grade 2 (67.8\% versus $76.9 \%$ versus $74.2 \%$ versus $94.1 \%$; $P=0.005)$ or grade $3(57.4 \%$ versus $65.7 \%$ versus $65.1 \%$ versus $77.1 \%$; $P=0.014$ ) disease. When removing DCIS patients, significant racial survival differences were still observed of those $<65$ years $(P<0.001)$ and $\geq 65$ years of age $(P=0.002)$, married $(P=0.002)$, with $\mathrm{ER}+/ \mathrm{PR}+$ disease $(P=0.001)$, undergoing modified radical mastectomy $(P<0.001)$, undergoing lymph node dissection $(P<0.001)$, undergoing adjuvant RT $(P=0.003)$, with nodal metastases $(P<0.001)$, with stage II $(P=0.005)$ or stage III $(P=0.005)$ disease, and with grade $2(P=0.005)$ or grade 3 $(P=0.016)$ disease.

Significant survival differences were also observed when DSS was analyzed (Table 4). Overall, Black patients had worse 5 -year DSS when compared to Whites, Hispanics, and Asians (66.3\% versus $73.8 \%$ versus $74.0 \%$ versus $85.3 \% ; P<0.001$ ). Significantly worse survival for Black patients was also seen in those $<65$ years of age $(P<0.001), \geq 65$ years $(P=$ $0.011)$, with invasive ductal carcinoma $(P<0.001)$, with $\mathrm{ER}+/ \mathrm{PR}+$ disease $(P=0.006)$, undergoing modified radical mastectomy $(P=0.002)$, receiving lymph node dissection $(P<0.001)$, undergoing adjuvant radiotherapy $(P=0.003)$, with nodal metastasis $(P<0.001)$, with stage II $(P=0.005)$ and stage III $(P=0.005)$ disease, and with grade II $(P=$ $0.005)$ and grade III $(P=0.014)$ disease when compared to the other races.

3.3. Multivariate Analysis. On Cox regression multivariate analysis, Black race $(P<0.001)$, older age at diagnosis
$(P<0.001)$, more advanced $\mathrm{T}$ stage $(P<0.001)$, more advanced stage of disease $(P<0.001)$, and higher tumor grade $(P<0.001)$ were independent prognostic factors for worse OS. Later year of diagnosis $(P=0.024)$ and undergoing lymph node dissection $(P<0.001)$ were significant predictors for better OS. Black race remained an independent prognostic factor for worse OS even after adjusting for the other stated factors (Table 5).

\section{Discussion}

4.1. Impact of Race. Our current study on male adults across the U.S. diagnosed from 1988 to 2010 with MBC demonstrated that race remains a significant prognostic factor. Specifically, Blacks were shown to have significantly worse OS compared to other races, which may be due in part to more advanced disease presentation. Interestingly, we found that there was no significant difference with regard to receipt of cancer-directed primary treatment by race after stratifying by stage of disease. However, Blacks were less likely to undergo lymph node dissection when compared to the other races.

Similar to our findings, O'Malley et al. showed in a retrospective study on 1,979 MBC patients that 5-year survival for Black MBC patients was significantly worse compared to other races [11]. In those 65 years or older with MBC, Crew et al. also showed that Black race was associated with increased cancer-specific mortality when compared to White men $(\mathrm{HR}=3.29,95 \% \mathrm{CI}=1.10-9.86)[10]$. Our report confirmed this survival disparity between races in MBC and revealed that it persists even after adjusting for age at diagnosis, year of diagnosis, primary treatment, stage of disease, and tumor grade. In contrast, a retrospective study on MBC cases in the Detroit metropolitan area by Simon et al. did not find race to be a predictor for survival [7]. These authors showed that Blacks $(1.42,95 \% \mathrm{CI}=1.07-1.77)$ had higher age-adjusted incidence rates of $\mathrm{MBC}$ per 100,000 compared to Whites $(0.88,95 \% \mathrm{CI}=0.86-1.00)$, but that race was not a significant prognostic factor for survival (relative risk $=1.18,95 \% \mathrm{CI}=$ $0.77-1.82$ ). Another study on six hundred patients from the California Cancer Registry did not observe any significant differences in outcome by race when comparing Blacks to Whites ( $\mathrm{HR}=1.32,95 \% \mathrm{CI}=0.75-2.33)$ after adjusting for age at diagnosis and stage of disease [8].

Several possibilities exist for the basis of our finding of racial disparity in OS. Previous studies have shown that Blacks have higher incidence rates of large tumor size, nodal metastases, high tumor grade, and negative hormone receptor expression compared to Whites $[8,9,11]$. These results are in concordance with our findings of more advanced disease presentation in Blacks compared to other racial groups. There also exists the prospect that Blacks have suboptimal access to specialist care. Crew et al. found that, in those 65 years of age or older, Black men were about half as likely to be seen by a medical oncologist for initial consult and receive adjuvant chemotherapy when compared to Whites, though this did not reach statistical significance [10]. A retrospective study among 9,630 women, 66 years of age or older and with early-stage breast cancer, showed that those 


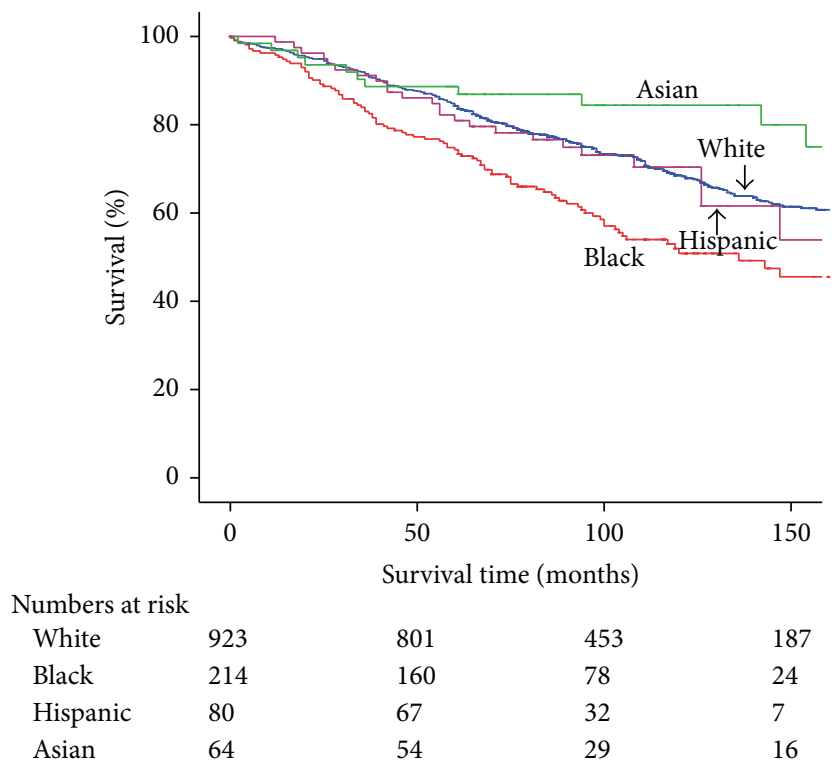

(a)

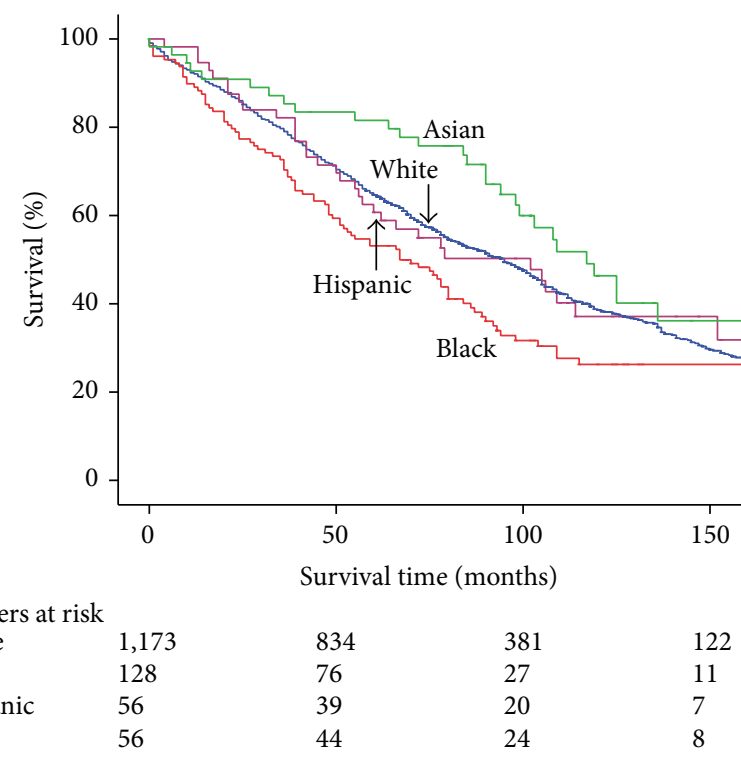

(b)

Figure 2: (a) Kaplan-Meier overall survival by race: $<65$ years. (b) Kaplan-Meier overall survival by race: $\geq 65$ years.

who saw a medical oncologist before surgery were more likely to undergo definitive surgery and axillary lymph node dissection [16]. Speculatively, this may have contributed to the lower rates of lymph node dissection seen in Blacks in our study. However, the SEER database lacks information on provider specialty, and thus, we are unable to determine if this had an effect in our analysis.

Differences in receipt of treatment between races have also been investigated in previous studies [7]. The majority of patients undergo modified radical mastectomy [11], and previous studies have shown no difference in local recurrence or survival rates in comparison to radical mastectomy [17]. Additionally, a previous retrospective study by Fogh et al. showed breast conservation surgery to have comparable rates of local control, disease-free survival, and overall survival with modified radical mastectomy or total simple mastectomy [18]. Simon et al. found no significant differences in the surgical treatment or administration of RT between Black and White males [7]. Similarly, we found that there was no significant difference between racial groups with regard to receipt of primary cancer-directed treatment when stratified by stage of disease. However, we did find that Blacks were less likely to undergo lymph node dissection. In a multi-institutional retrospective study on 397 men with nonmetastatic breast cancer, Cutuli et al. showed that men who undergo nodal dissection may derive some benefit in outcome. These authors showed that regional nodal recurrence occurred in only $1.2 \%$ of patients who underwent axillary dissection compared to $13 \%$ in those without dissection $(P<0.001)$ [19]. However, the SEER database does not record disease recurrence. Finally, we did not note a difference in receipt of adjuvant RT between racial groups. Though there are still limited data from small retrospective studies regarding the indications for postmastectomy RT in MBC and its impact on outcome [2022], a prior retrospective study by Fogh et al., on forty-two male nonmetastatic ER+/PR+ breast cancer patients, showed adjuvant RT and tamoxifen to be superior in terms of overall survival to adjuvant tamoxifen alone, adjuvant RT alone, and no adjuvant therapy, suggesting a possible role for adjuvant $\mathrm{RT}$ in addition to tamoxifen in ER+/PR+ MBC [23]. Thus, further studies are warranted to justify its use for this patient population.

In our analysis, age was divided by the median age of 65 years, and younger age was defined as those younger than 65 years of age. Black males were more likely to be diagnosed with breast cancer at a younger age compared to other races. Previous studies have shown that, in women younger than 35 years of age, Black patients were at increased risk for breast cancer compared to other races and that they have an increased mortality risk when compared with White women of the same age $[24,25]$. Black MBC patients in our study were also less likely to be married compared to other races. Unmarried patients in our analysis were shown to have worse outcome, and there certainly exists a possibility of a relation between the respective associations of younger age and being nonmarried for Black patients. Interestingly, a recent report by the U.S. Bureau of Labor Statistics has shown that Blacks married at a later age and at lower rates when compared with Whites [26].

Black MBC patients were significantly more likely to have ER-/PR- disease (5.3\% versus $4.0 \%, P<0.001)$ and less likely to have $\mathrm{ER}+/ \mathrm{PR}+$ disease $(56.0 \%$ versus $64.8 \%, P<$ $0.001)$ when compared to White patients. These findings are in concordance with previous studies showing an increased association of triple-negative breast cancer in Black female breast cancer patients when compared to other races [27]. 


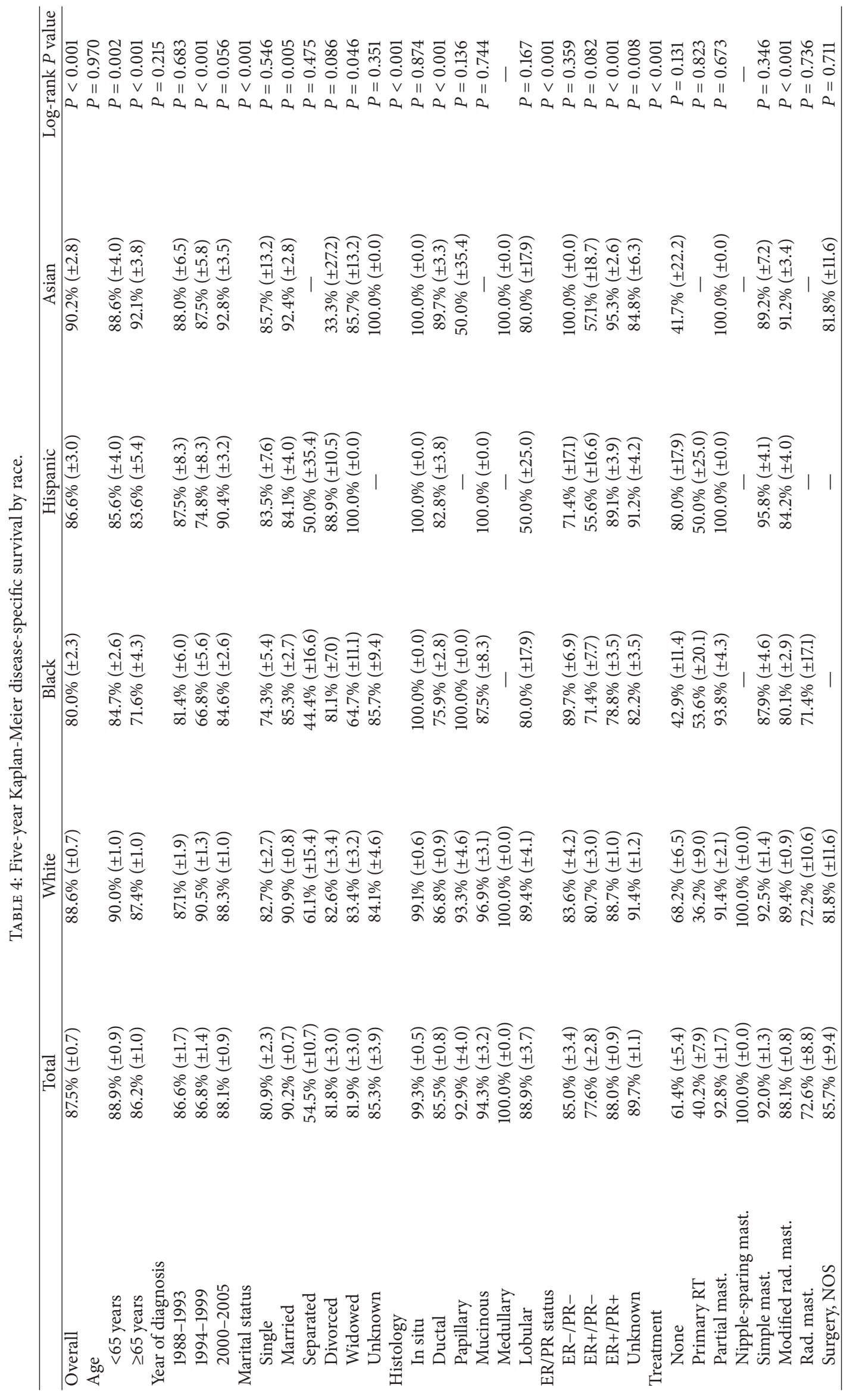




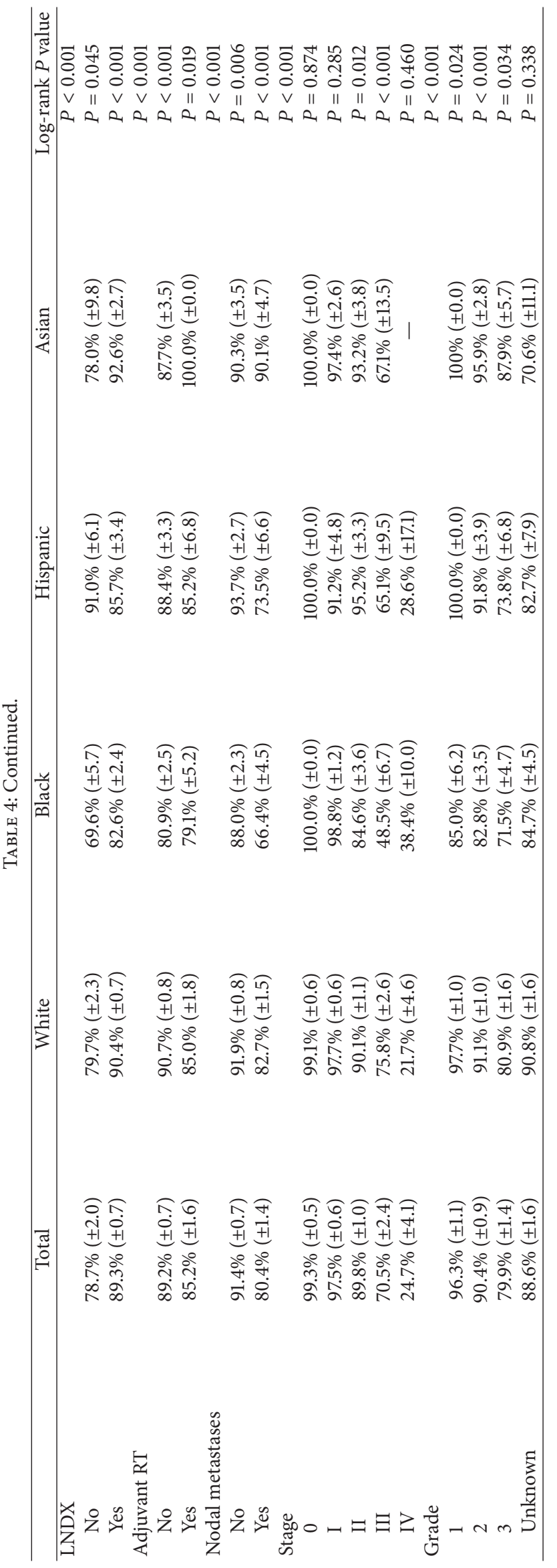


TABLE 5: Cox regression multivariate analysis.

\begin{tabular}{lccc}
\hline Prognostic factor & Hazard ratio & 95\% confidence interval & $P$ value \\
\hline Race $^{\mathrm{a}}$ & 1.386 & $1.185-1.621$ & $P<0.001$ \\
Age at diagnosis $^{\mathrm{b}}$ & 1.049 & $1.044-1.054$ & $P<0.001$ \\
Year of diagnosis $^{\mathrm{b}}$ & 0.983 & $0.971-0.994$ & $P=0.004$ \\
Lymph node dissection $^{\mathrm{c}}$ & 0.581 & $0.503-0.671$ & $P<0.001$ \\
T stage $^{\mathrm{d}}$ & 1.452 & $1.315-1.603$ & $P<0.001$ \\
Stage of disease $^{\mathrm{e}}$ & 1.203 & $1.114-1.300$ & $P<0.001$ \\
Grade $^{\mathrm{f}}$ & 1.237 & $1.134-1.349$ & $P<0.001$ \\
\hline
\end{tabular}

${ }^{a}$ Black versus non-Black.

${ }^{\mathrm{b}}$ Continuous.

${ }^{\mathrm{c}}$ Yes versus no.

${ }^{\mathrm{d}}$ Metastatic disease versus 4 versus 3 versus 2 versus 1 versus in situ.

${ }^{\mathrm{e}} \mathrm{IV}$ versus III versus II versus I versus 0 .

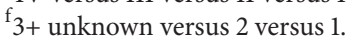

A higher proportion of ER-/PR- disease observed in Black $\mathrm{MBC}$ patients compared to Whites may be a contributing factor to their poorer outcome. Prior investigations have shown triple-negative breast cancer to be associated with poorer outcome $[28,29]$; however, a limitation of the SEER database is that HER2 status, which has been associated with outcome in female breast cancer patients, is not recorded [30].

An interesting finding from our study is the significantly better OS observed in Asian MBC patients compared to the other races of those $\geq 65$ years $(P=0.011)$, married $(P=$ $0.037)$, with invasive ductal carcinoma $(P<0.001)$, with $\mathrm{ER}+/ \mathrm{PR}+$ disease $(P=0.006)$, receiving modified radical mastectomy $(P=0.002)$, undergoing lymph node dissection $(P<0.001)$, with nodal metastasis $(P<0.001)$, and with grade $2-3$ disease $(P=0.005, P=0.014)$. A previous study on prostate cancer patients showed that Japanese men had significantly better survival than White patients; however, the reasons were unclear [31]. In advanced stage, non-small cell lung cancer patients, Asians were found to have significantly higher survival rates and greater response rates to chemotherapy [32]. In a study on female breast cancer patients, Japanese women were also found to have significantly better survival compared to White patients, but there were no significant differences between Chinese, Filipino, and White women [33]. In our study, they were more likely to present with earlier stage disease $(P=0.001)$, and these results are similar to those seen in previous studies for other cancer sites [34]. Though Asian MBC patients make up only a small percentage of those with breast cancer, further studies are warranted to investigate possible etiologies for differences in outcome and the heterogeneity observed in this fast-growing population in the United States [35].

4.2. Prognostic Factors. A previous retrospective study on $2,537 \mathrm{MBC}$ patients showed that age $\geq 65$ years $(\mathrm{HR}=1.59)$, tumor size $2-5 \mathrm{~cm}(\mathrm{HR}=1.40)$, and nodal metastases $(\mathrm{HR}=$ 1.50) were independent prognostic factors for worse survival [2]. In a single-institutional study on twenty-nine $\mathrm{MBC}$ patients, Moore et al. also showed that older age was a significant predictor for worse OS $(P=0.008)$ [4]. Indeed, we found that, in addition to Black race, older age at diagnosis, earlier year of diagnosis, not undergoing mastectomy, more advanced stage of disease, and higher tumor grade portended for worse OS on multivariate analysis.

The majority of new cancers and cancer deaths have been shown to occur in those 65 years of age or older [36], and our analysis showed that those $>65$ years of age had worse OS. Previous studies have shown older age to be associated with chronic illness and age-related health conditions which can have adverse impact on quality of life, rates of disability, and independent living $[37,38]$. The preexisting health conditions of a patient may have an impact on clinical decision making and cancer treatment. Appropriate assessment and management of preexisting comorbidities can potentially provide benefit, enhance quality of life, and, subsequently, impact patient outcome.

Our study cohort, consisting of patients from 1988 to 2010, showed an improvement in 5-year OS over time. We observed a significant improvement in 5-year OS for all MBC patients over the time periods 1988-1993, 1994-1999, and 2000-2005 (68.9\% versus $72.0 \%$ versus $75.2 \% ; P=0.018$ ). Similarly, Anderson et al. showed that hazard ratios in MBC patients fell by $28 \%$ from the 1976-1985 time period to the 1996-2005 period $(P=0.03)$ [3]. Year of diagnosis was not shown to affect survival in a prior retrospective study; however, this report was limited to patients diagnosed in 1997 or earlier [11]. There exist several possible factors for our finding, including more advanced treatments and improved multidisciplinary approach to cancer care with time.

A report by Aizer et al. found that unmarried patients were at significant risk for suboptimal management, metastatic disease, and poor outcome in a study on over one million patients diagnosed with lung, breast, prostate, colorectal, liver/intrahepatic bile duct, pancreatic, non-Hodgkin lymphoma, head/neck, ovarian, or esophageal cancer [39]. On univariate analysis, our report on MBC showed that those who were married had the highest 5-year OS of any marital status subgroup. However, marital status was not found to be a significant prognostic factor in our multivariate analysis. Nonetheless, there is now awareness that those unmarried may have poorer outcomes and that social support services 
have the potential to have a positive impact on treatment adherence, response, and survival.

To our knowledge, this report is the largest comprehensive study on $\mathrm{MBC}$ to examine the impact of race on outcome, as well as prognostic factors for survival in these patients. The strength in our study lies in the large patient numbers from four racial groups, White, Black, Hispanic, and Asian, which allowed for statistical analysis powered for detection of differences in treatment and outcome and investigation of different prognostic factors in multivariate analysis. Prior studies examining race in $\mathrm{MBC}$ have been limited by either geographic distribution $[7,8]$, age at diagnosis [10], or analyzed much earlier time periods [11]. In contrast, this SEER population-based cancer analysis is broadly representative of the U.S. population, covering approximately 28 percent of the population, thus decreasing selection bias risks that may be associated with smaller analyses [15]. However, our current study also has several limitations including no record on centralized pathology review, margin status after primary surgery, adjuvant systemic therapy, disease recurrence, and information on risk factors for MBC such as first-degree relative with breast cancer, BRCA status, hormone levels, body mass index, and obesity $[40,41]$. In addition, our analysis lacked information on cultural, socioeconomic, and behavioral factors which may have an effect on outcome. Thus, we cannot evaluate the extent to which these factors may contribute to racial disparities.

4.3. Conclusions. In summary, the results of this SEER analysis of 4,279 MBC patients showed that race, age at diagnosis, year of diagnosis, $\mathrm{T}$ stage, stage of disease, and tumor grade are independent predictors for survival. Blacks were found to have an overall survival disadvantage compared to Whites, Hispanics, and Asians, which may be in part due to differences in disease presentation. Our study did show that Blacks were less likely to receive lymph node dissection of which patients may derive benefit, though we did not observe receipt of primary treatment, after stratifying for stage of disease, to be an underlying factor contributing to this disparity in survival outcome. Further studies are warranted to investigate if racial disparities in $\mathrm{MBC}$ are associated with socioeconomic status, access to medical care, limiting medical comorbidities, and genetic and biologic etiologies.

\section{Conflict of Interests}

The authors declare that there is no conflict of interests regarding the publication of this paper.

\section{References}

[1] R. Siegel, J. Ma, Z. Zou, and A. Jemal, "Cancer statistics, 2014," CA Cancer Journal for Clinicians, vol. 64, no. 1, pp. 9-29, 2014.

[2] S. H. Giordano, D. S. Cohen, A. U. Buzdar, G. Perkins, and G. N. Hortobagyi, "Breast carcinoma in men: a population-based study," Cancer, vol. 101, no. 1, pp. 51-57, 2004.

[3] W. F. Anderson, I. Jatoi, J. Tse, and P. S. Rosenberg, "Male breast cancer: a population-based comparison with female breast cancer," Journal of Clinical Oncology, vol. 28, no. 2, pp. 232-239, 2010.

[4] J. Moore, M. I. Friedman, T. Gansler et al., "Prognostic indicators in male breast carcinoma," Breast Journal, vol. 4, no. 4, pp. 261-269, 1998.

[5] V. F. Guinee, H. Olsson, T. Moller et al., "The prognosis of breast cancer in males. A report of 335 cases," Cancer, vol. 71, pp. 154161, 1993.

[6] J. Vetto, S. Y. Jun, D. Paduch et al., "Stages at presentation, prognostic factors, and outcome of breast cancer in males," The American Journal of Surgery, vol. 177, pp. 379-383, 1999.

[7] M. S. Simon, E. McKnight, A. Schwartz, S. Martino, and G. M. Swanson, "Racial differences in cancer of the male breast15 year experience in the Detroit Metropolitan Area," Breast Cancer Research and Treatment, vol. 21, no. 1, pp. 55-62, 1992.

[8] M. Chavez-Macgregor, C. A. Clarke, D. Lichtensztajn, G. N. Hortobagyi, and S. H. Giordano, "Male breast cancer according to tumor subtype and race: a population-based study," Cancer, vol. 119, no. 9, pp. 1611-1617, 2013.

[9] W. F. Anderson, M. D. Althuis, L. A. Brinton, and S. S. Devesa, "Is male breast cancer similar or different than female breast cancer?" Breast Cancer Research and Treatment, vol. 83, no. 1, pp. 77-86, 2004.

[10] K. D. Crew, A. I. Neugut, X. Wang et al., "Racial disparities in treatment and survival of male breast cancer," Journal of Clinical Oncology, vol. 25, no. 9, pp. 1089-1098, 2007.

[11] C. D. O’Malley, A. W. Prehn, S. J. Shema, and S. L. Glaser, "Racial/ethnic differences in survival rates in a populationbased series of men with breast carcinoma," Cancer, vol. 94, no. 11, pp. 2836-2843, 2002.

[12] M. S. Simon, A. G. Schwartz, S. Martino, and G. M. Swanson, "Trends in the diagnosis in situ breast cancer in the Detroit Metropolitan area, 1973 to 1987," Cancer, vol. 69, no. 2, pp. 466469, 1992.

[13] A. Dehal, A. Abbas, and S. Johna, "Racial disparities in clinical presentation, surgical treatment and in-hospital outcomes of women with breast cancer: analysis of nationwide inpatient sample database," Breast Cancer Research and Treatment, vol. 139, no. 2, pp. 561-569, 2013.

[14] Z. A. Nahleh, R. Srikantiah, M. Safa, A.-R. Jazieh, A. Muhleman, and R. Komrokji, "Male breast cancer in the veterans affairs population: a comparative analysis," Cancer, vol. 109, no. 8, pp. 1471-1477, 2007.

[15] "Surveillance, Epidemiology, and End Results (SEER) Program," National Cancer Institute, http://www.seer.cancer.gov.

[16] N. L. Keating, M. B. Landrum, J. Z. Ayanian, E. P. Winer, and E. Guadagnoli, "Consultation with a medical oncologist before surgery and type of surgery among elderly women with earlystage breast cancer," Journal of Clinical Oncology, vol. 21, no. 24, pp. 4532-4539, 2003.

[17] A. G. Digenis, C. B. Ross, J. G. Morrison, G. W. Holcomb III, and V. H. Reynolds, "Carcinoma of the male breast: a review of 41 cases," Southern Medical Journal, vol. 83, no. 10, pp. 1162-1167, 1990.

[18] S. Fogh, L. A. Kachnic, S. I. Goldberg, A. G. Taghian, S. N. Powell, and A. E. Hirsch, "Localized therapy for male breast cancer: functional advantages with comparable outcomes using breast conservation," Clinical Breast Cancer, vol. 13, no. 5, pp. 344-349, 2013.

[19] B. Cutuli, M. Lacroze, J. M. Dilhuydy et al., "Male breast cancer: results of the treatments and prognostic factors in 397 cases," 
European Journal of Cancer A: General Topics, vol. 31, no. 12, pp. 1960-1964, 1995.

[20] R. A. Spence, G. MacKenzie, J. R. Anderson et al., "Long-term survival following cancer of the male breast in Northern Ireland. A report of 81 cases," Cancer, vol. 55, pp. 648-652, 1985.

[21] C. Erlichman, K. C. Murphy, and T. Elhakim, "Male breast cancer: a 13-year review of 89 patients," Journal of Clinical Oncology, vol. 2, no. 8, pp. 903-909, 1984.

[22] U. Schuchardt, M. H. Seegenschmiedt, M. J. Kirschner, H. Renner, and R. Sauer, "Adjuvant radiotherapy for breast carcinoma in men: a 20-year clinical experience," The American Journal of Clinical Oncology: Cancer Clinical Trials, vol. 19, no. 4, pp. 330336, 1996.

[23] S. Fogh, A. E. Hirsch, J. P. Langmead et al., "Use of tamoxifen with postsurgical irradiation may improve survival in estrogen and progesterone receptor-positive male breast cancer," Clinical Breast Cancer, vol. 11, no. 1, pp. 39-45, 2011.

[24] M. D. Althuis, D. D. Brogan, R. J. Coates et al., "Breast cancers among very young premenopausal women (United States)," Cancer Causes and Control, vol. 14, no. 2, pp. 151-160, 2003.

[25] L. A. Newman, S. Bunner, K. Carolin et al., "Ethnicity related differences in the survival of young breast carcinoma patients," Cancer, vol. 95, no. 1, pp. 21-27, 2002.

[26] US Bureau of Labor Statistics, "Marriage and divorce: patterns by gender, race, and educational attainment," 2013, http:// www.bls.gov/opub/mlr/2013/article/pdf/marriage-and-divorcepatterns-by-gender-race-and-educational-attainment.pdf.

[27] L. A. Stead, T. L. Lash, J. E. Sobieraj et al., “Triple-negative breast cancers are increased in black women regardless of age or body mass index," Breast Cancer Research, vol. 11, no. 2, article R18, 2009.

[28] B. G. Haffty, Q. Yang, M. Reiss et al., "Locoregional relapse and distant metastasis in conservatively managed triple negative early-stage breast cancer," Journal of Clinical Oncology, vol. 24, no. 36, pp. 5652-5657, 2006.

[29] K. R. Bauer, M. Brown, R. D. Cress, C. A. Parise, and V. Caggiano, "Descriptive analysis of estrogen receptor (ER)-negative, progesterone receptor (PR)-negative, and HER2-negative invasive breast cancer, the so-called triple-negative phenotype: a population-based study from the California Cancer Registry," Cancer, vol. 109, no. 9, pp. 1721-1728, 2007.

[30] R. Seshadri, F. A. Firgaira, D. J. Horsfall, K. McCaul, V. Setlur, and P. Kitchen, "Clinical significance of HER-2/neu oncogene amplification in primary breast cancer," Journal of Clinical Oncology, vol. 11, no. 10, pp. 1936-1942, 1993.

[31] A. S. Robbins, T. M. Koppie, S. L. Gomez, A. Parikh-Patel, and P. K. Mills, "Differences in prognostic factors and survival among white and Asian men with prostate cancer, California, 19952004," Cancer, vol. 110, no. 6, pp. 1255-1263, 2007.

[32] R. A. Soo, M. Loh, T. S. Mok et al., "Ethnic differences in survival outcome in patients with advanced stage non-small cell lung cancer: results of a meta-analysis of randomized controlled trials," Journal of Thoracic Oncology, vol. 6, no. 6, pp. 1030-1038, 2011.

[33] M. D. Pineda, E. White, A. R. Kristal, and V. Taylor, "Asian breast cancer survival in the US: a comparison between Asian immigrants, US-born Asian Americans and Caucasians," International Journal of Epidemiology, vol. 30, no. 5, pp. 976-982, 2001.

[34] J. K. Chan, K. Fuh, J. Y. Shin et al., "The treatment and outcomes of early-stage epithelial ovarian cancer: have we made any progress?" British Journal of Cancer, vol. 98, no. 7, pp. 1191-1196, 2008.

[35] United States Census Bureau, The Asian Population: 2010, 2012, http://www.census.gov/prod/cen2010/briefs/c2010br-11.pdf.

[36] L. A. G. Ries, D. Melbert, M. Krapcho et al., SEER Cancer Statistics Review, 1975-2004, National Cancer Institute, Bethesda, Md, USA, 2007.

[37] K. M. Bellizzi and J. H. Rowland, "Role of comorbidity, symptoms and age in the health of older survivors following treatment for cancer," Aging Health, vol. 3, no. 5, pp. 625-635, 2007.

[38] A. V. Rao and W. Demark-Wahnefried, "The older cancer survivor," Critical Reviews in Oncology/Hematology, vol. 60, no. 2, pp. 131-143, 2006.

[39] A. A. Aizer, M.-H. Chen, E. P. McCarthy et al., "Marital status and survival in patients with cancer," Journal of Clinical Oncology, vol. 31, no. 31, pp. 3869-3876, 2013.

[40] Y. C. Tai, S. Domchek, G. Parmigiani, and S. Chen, "Breast cancer risk among male BRCA1 and BRCA2 mutation carriers," Journal of the National Cancer Institute, vol. 99, no. 23, pp. 18111814, 2007.

[41] L. A. Brinton, D. A. Richesson, G. L. Gierach et al., "Prospective evaluation of risk factors for male breast cancer," Journal of the National Cancer Institute, vol. 100, no. 20, pp. 1477-1481, 2008. 


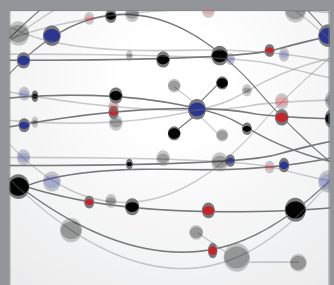

The Scientific World Journal
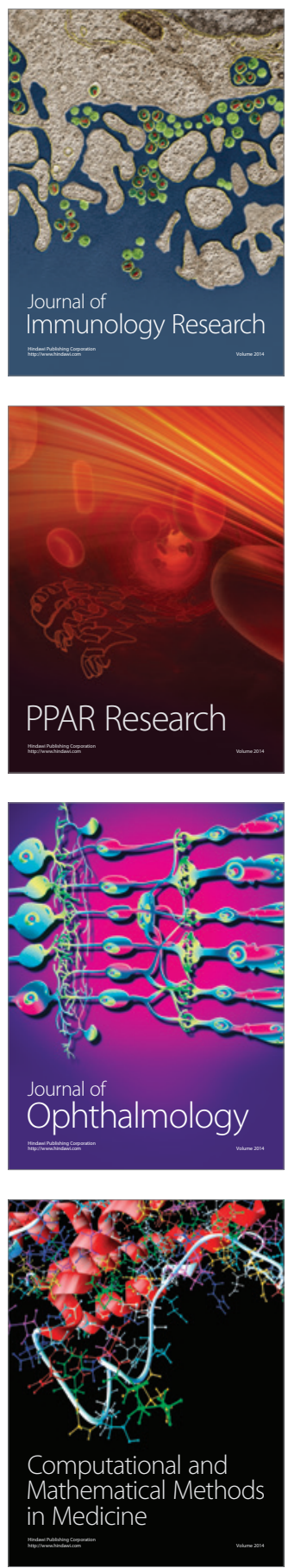

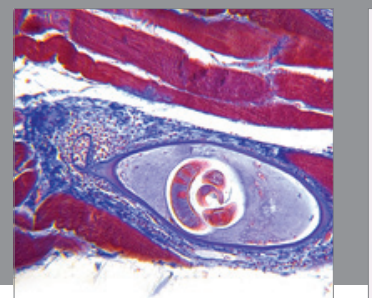

Gastroenterology

Research and Practice
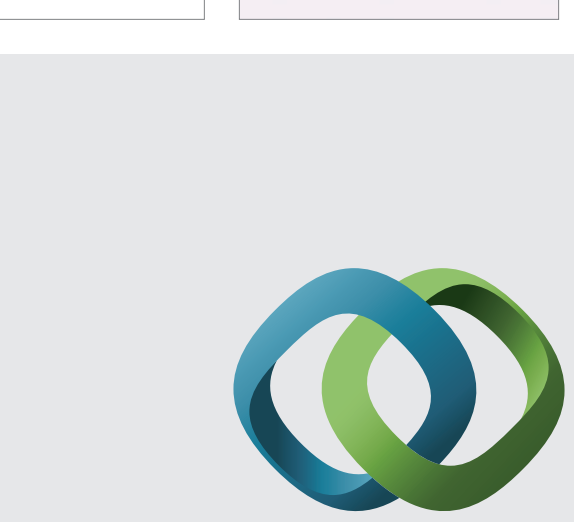

\section{Hindawi}

Submit your manuscripts at

http://www.hindawi.com
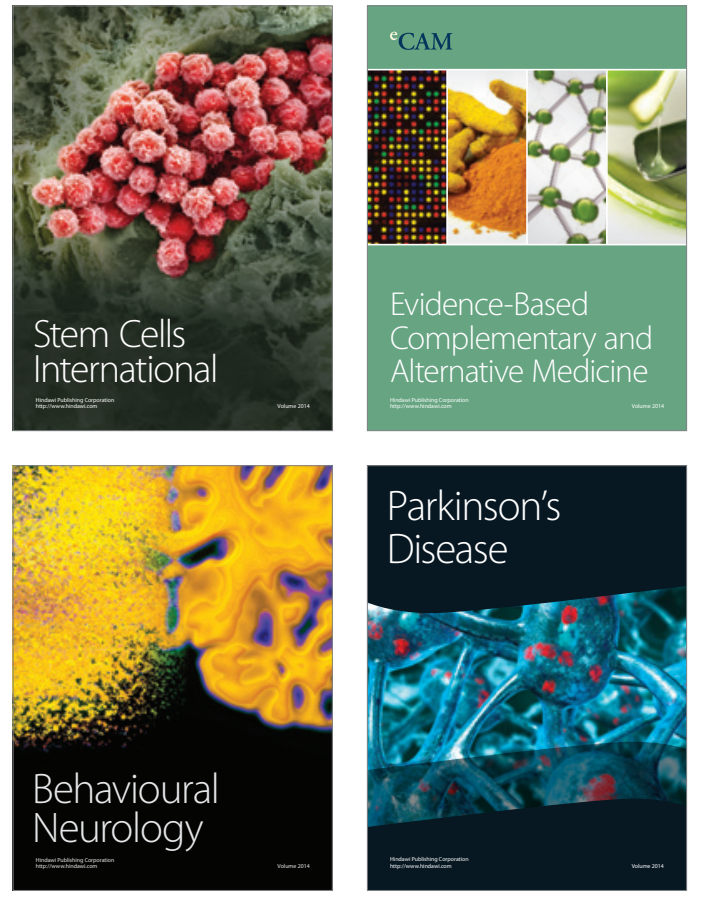
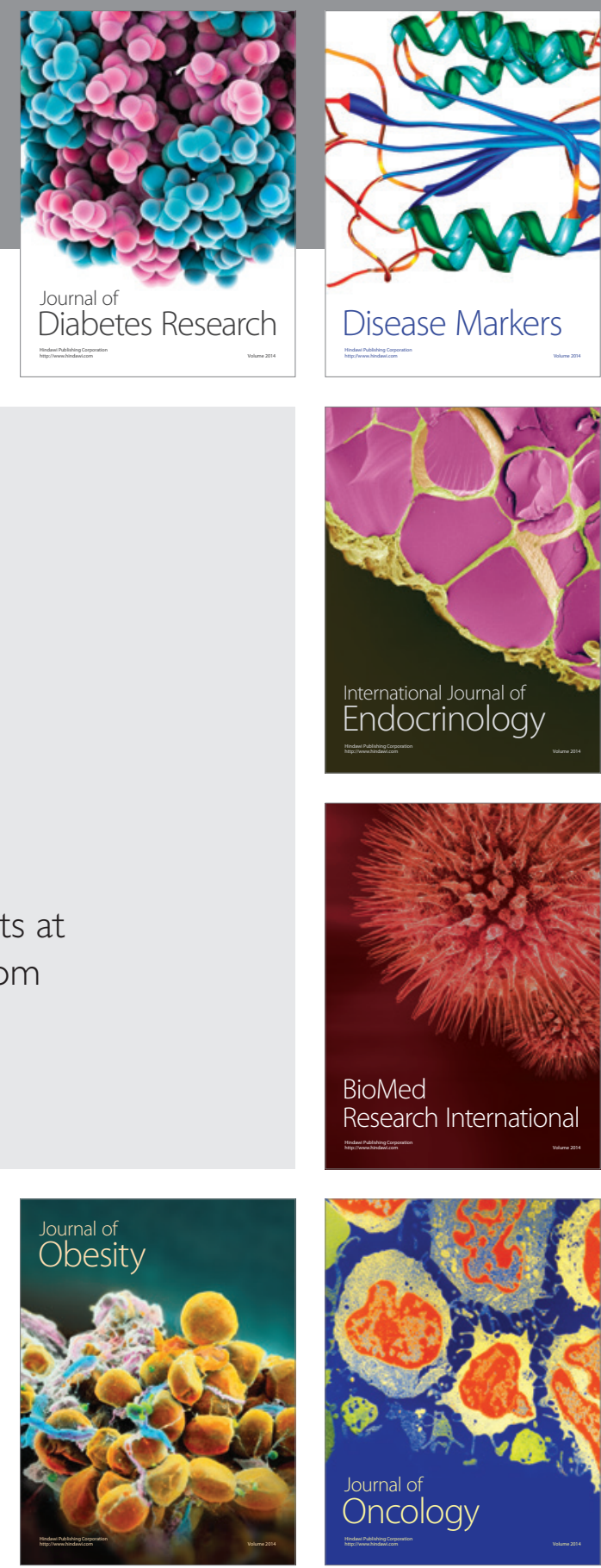

Disease Markers
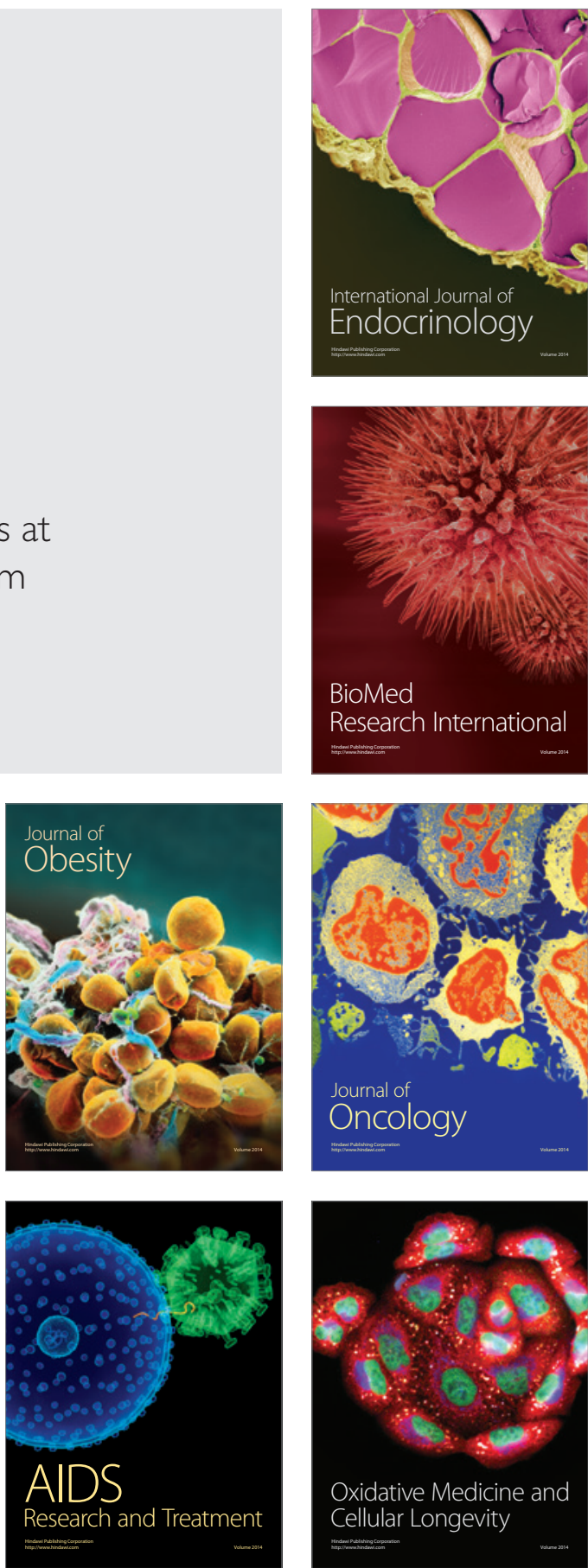